\title{
Effect of the continuous intake of probiotic-fermented milk containing Lactobacillus casei strain Shirota on fever in a mass outbreak of norovirus gastroenteritis and the faecal microflora in a health service facility for the aged
}

Satoru Nagata ${ }^{1}$, Takashi Asahara ${ }^{2}$, Toshihisa Ohta ${ }^{2}$, Toshihiko Yamada ${ }^{3,4}$, Shigemi Kondo ${ }^{4}$, Lei Bian ${ }^{1}$, Chongxin Wang ${ }^{1}$, Yuichiro Yamashiro ${ }^{1}$ and Koji Nomoto ${ }^{2}$

${ }^{1}$ Department of Laboratory for Probiotic Research, Juntendo University, 2-1-1 Hongo, Bunkyo-ku, Tokyo 113-8421, Japan

${ }^{2}$ Yakult Central Institute for Microbiological Research, Tokyo, Japan

${ }^{3}$ Yuyu, Health Service Facility for Aged, Ichikawa City, Japan

${ }^{4}$ Department of Clinical Laboratory Medicine, Juntendo University School of Medicine, Tokyo, Japan

(Received 29 June 2010 - Revised 18 October 2010 - Accepted 9 November 2010 - First published online 27 April 2011)

\section{Abstract}

For conducting effective risk management in long-stay elderly people at a health service facility, we performed an open case-controlled study to evaluate the effect of the intake of probiotic-fermented milk containing Lactobacillus casei strain Shirota (LcS-fermented milk) on norovirus gastroenteritis occurring in the winter season during the intake period. A total of seventy-seven elderly people (mean age 84 years) were enrolled in the study. During a 1-month period, there was no significant difference in the incidence of norovirus gastroenteritis between the LCS-fermented milk-administered $(n$ 39) and the non-administered ( $n$ 38) groups; however, the mean duration of fever of $>37^{\circ} \mathrm{C}$ after the onset of gastroenteritis was 1.5 (SD 1.7) $\mathrm{d}$ in the former and 2.9 (SD 2.3) $\mathrm{d}$ in the latter group, showing a significant shortening in the former group $(P<0 \cdot 05)$. RT-quantitative PCR analysis targeting ribosomal RNA showed both Bifidobacterium and Lactobacillus to be significantly dominant, whereas Enterobacteriaceae decreased in faecal samples from the administered group ( $n$ 10, mean age 83 years), with a significant increase in faecal acetic acid concentration. Continuous intake of LcS-fermented milk could positively contribute to the alleviation of fever caused by norovirus gastroenteritis by correcting the imbalance of the intestinal microflora peculiar to the elderly, although such consumption could not protect them from the disease.

Key words: Probiotic-fermented milk: Norovirus gastroenteritis: Probiotics: Fever: Faecal microflora: Organic acids: Long-stay elderly people

Elderly persons are especially prone to infection, as many physiological and immune responses as well as organ functions decline with age. An infection under these conditions tends to be refractory and becomes severe. In elder care facilities, it is highly likely that an infection, after it breaks out, will spread easily among residents. It has therefore become a major challenge for residential long-term care homes for the elderly to take measures to prevent infections among their elderly residents.

Probiotics are defined as, live microorganisms which when administered in adequate amounts confer a health benefit on the host' ${ }^{(1)}$. Lactobacillus casei strain Shirota (LcS) is the most common probiotic bacterium and is clinically proven in many reports to have an immuno-enhancing effect $^{(2)}$, protective effects against post-operative infectious complications $^{(3)}$, cancer $^{(4)}$ and allergies ${ }^{(5)}$, and to regulate intestinal function ${ }^{(6)}$.

In this case, for the purposes of risk factor control among elderly residents of residential long-term care homes, we introduced a probiotic-fermented milk containing LcS (LcSfermented milk). We examined the effects of LcS-fermented milk intake on infectious gastroenteritis caused by various norovirus outbreaks in the winter (December 2006) during the LcS-fermented milk trial period, with exoergic reactions being referred to as an indicator. The effects of LcS-fermented milk intake on the intestinal flora and intestinal environment of the elderly residents of nursing homes were examined and are reported in the present study.

Abbreviation: LcS, Lactobacillus casei strain Shirota.

*Corresponding author: Dr S. Nagata, fax +8135689 0082, email snagata@juntendo.ac.jp 


\section{Materials and methods}

\section{Human subjects}

Effects on norovirus gastroenteritis. A total of seventy-seven frail elderly residents of 'Yuyu' Municipal Health Service Facility for the Aged in the city of Ichikawa, who had been admitted to the facility before December 2006 with the intention of eventually returning home, were enrolled in the trial of LCS-fermented milk (age 84 (SD 9) years; fifty male and twentyseven female residents).

Effects on the composition of faecal flora. A total of ten frail, long-term elderly residents of the facility, who had been admitted before April 2007, were enrolled in the trial of LcS-fermented milk (age 83 (SD 10) years; three male and seven female residents).

The trial was performed in compliance with the principles of the Helsinki Declaration (adopted in 1964; amended in 1975, 1983, 1989, 1996 and 2000) and approved by the Clinical Research Ethics Committee of Juntendo University Hospital (Tokyo, Japan), whereby a thorough explanation was given to the human subjects before the trial as to the purpose and method of the trial in order to obtain their written consent. Any applicable residents who were unable to consume the test diet properly due to dysphagia or for other medical reasons were excluded.

\section{Test diet}

The test diet consisted of fermented milk containing LcS (YIT9029, LcS) at $4 \times 10^{10}$ cells $/ 80 \mathrm{ml}$ bottle according to a previous report ${ }^{(2,7)}$. LcS-fermented milk is made from sugar, skimmed milk, high-fructose corn syrup and flavour ingredients. The fermented milk providing $259 \cdot 4 \mathrm{~kJ}(62 \mathrm{kcal})$ contains $1.0 \mathrm{~g}$ protein, $0.1 \mathrm{~g}$ fat, $14.4 \mathrm{~g}$ carbohydrate, $15 \mathrm{mg}$ $\mathrm{Na}$ and LCS at $4 \times 10^{10}$ cells or more in each bottle.

\section{Study schedule and ingestion of the test diet}

Effects on norovirus gastroenteritis. The trial was conducted as an open study, comparing the administered and nonadministered groups (Fig. 1). Of all residents who had given their written consent, thirty-nine who were residing on a certain floor were allocated to the LcS-fermented milkadministered group and thirty-eight residents on another floor were allocated to the non-administered group. The age and sex distribution were confirmed not to differ substantially between the two groups (Table 1). Having been restricted to the intake of a lactic acid bacteria-containing product for 2 weeks, the LcS-fermented milk-administered group was given one bottle of the test diet per day on a continuous basis from October 2006 until they were discharged from the facility. For the 1-month period from 1 to 31 December 2006, during the intake of LcS-fermented milk, the effects of continuous intake of the test diet were observed.

Effects on the composition of faecal flora. The trial was conducted as a controlled study, comparing the data before and after administration (Fig. 1). Of all residents who had given their written consent, ten who were residing on a certain floor were enrolled in the trial. From April to August 2007, after having been restricted to the intake of a lactic acid bacteria-containing product for 2 weeks, they were given one bottle of the test diet per day on a continuous basis.

Schedule 1 (norovirus gastroenteritis)

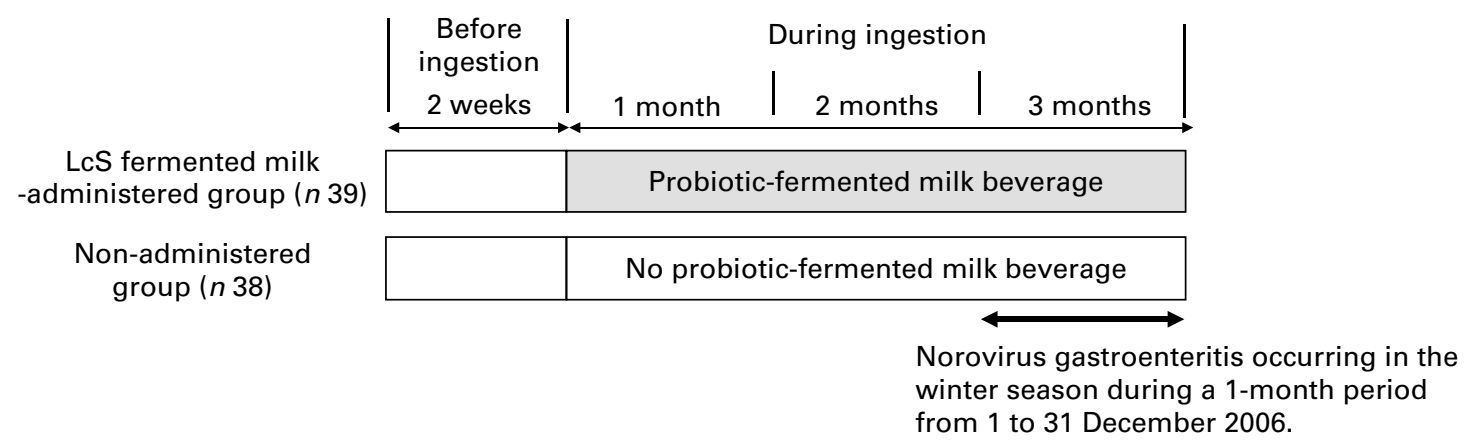

Schedule 2 (faecal microflora and organic acids)

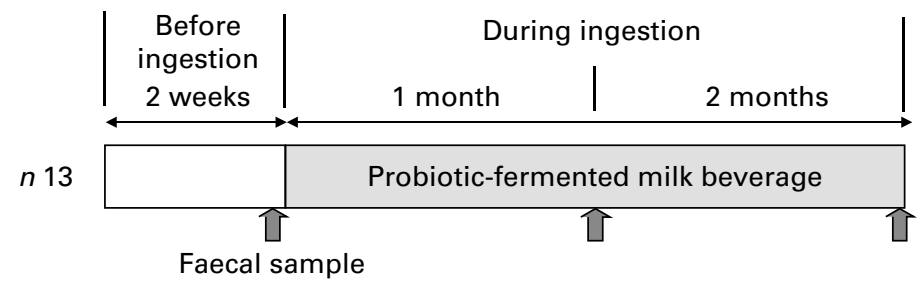

Fig. 1. Study design. LcS, Lactobacillus casei strain Shirota. 
Table 1. Duration of fever of $>37$ and $>38^{\circ} \mathrm{C}$ after the onset of norovirus gastroenteritis in December 2006 (Mean values, standard deviations and number of subjects)

\begin{tabular}{|c|c|c|c|c|c|}
\hline \multirow[b]{2}{*}{ Item } & \multicolumn{2}{|c|}{$\begin{array}{l}\text { Non-administered } \\
\text { group }\end{array}$} & \multicolumn{2}{|c|}{$\begin{array}{l}\text { LcS-fermented } \\
\text { milk-administered } \\
\text { group }\end{array}$} & \multirow[b]{2}{*}{$P^{*}$} \\
\hline & Mean & SD & Mean & SD & \\
\hline Number of subjects $(n)$ & \multicolumn{2}{|c|}{38} & \multicolumn{2}{|c|}{39} & \\
\hline \multicolumn{6}{|l|}{ Sex distribution $(n)$} \\
\hline Male & \multicolumn{2}{|c|}{5} & \multicolumn{2}{|c|}{8} & \\
\hline Female & \multicolumn{2}{|c|}{16} & \multicolumn{2}{|c|}{19} & \\
\hline Age (years) & 85 & 8 & 83 & 9 & NS \\
\hline Number of norovirus gastroenteritis cases $(n)$ & \multirow{2}{*}{\multicolumn{2}{|c|}{$21 \dagger$}} & \multirow{2}{*}{\multicolumn{2}{|c|}{$27 \dagger$}} & NS \\
\hline Duration of fever (d) & & & & & NS \\
\hline$>37^{\circ} \mathrm{C}$ & 2.9 & $2 \cdot 3$ & 1.5 & 1.7 & 0.027 \\
\hline$>38^{\circ} \mathrm{C}$ & 0.7 & 1.2 & 0.4 & 1.0 & 0.088 \\
\hline
\end{tabular}

The subjects were instructed not to change their daily diet and exercise programme at the facility, other than taking the test diet at a fixed time every day.

\section{Examination methods}

Survey of the medical record data. During the trial period, data from the subjects were recorded every day by nurses with regard to their faecal condition, frequency of bowel movements, axillary temperature, vomiting, medicine prescribed on a temporary basis, physical condition (health and sickness) and whether they had taken the test diet. As a precaution, they were instructed to generally eat only the food prepared by the facility and to restrict excessive intake of dairy products and oligosaccharide-containing products. No restriction was placed on medication. When any medicine was taken or when there was an excessive intake of dairy products, etc., this was recorded in the medical records.

Survey of norovirus gastroenteritis. With regard to the ten residents who suffered vomiting or diarrhoea during the trial and observation period (December 2006), faecal samples were taken to establish infectious gastroenteritis caused by norovirus using the Norovirus Antigen Detection Kit (NV-AD Seiken; Denka Seiken Company Limited, Osaka, Japan). The result was that some of these subjects were found to be positive. Thereafter, patients who suffered vomiting or diarrhoea during the trial and observation period were diagnosed to have infectious gastroenteritis (epidemiological clinical diagnosis).

\section{Faecal bacteriological examination}

Faecal sampling. A fresh faecal sample was taken from all residents of the facility before the test diet ingestion as well as at 1 and 2 months after ingestion according to a previous report ${ }^{(8)}$. Immediately after defecation, faecal samples were weighed and then suspended in nine volumes of RNAlater (Ambion, Inc., Austin, TX, USA), an RNA stabilisation solution, and the preparations were incubated for $10 \mathrm{~min}$ at room temperature.
Isolation of total RNA or total DNA. For RNA (or DNA) stabilisation, faecal homogenate $(200 \mu \mathrm{l})$ was added to $1 \mathrm{ml}$ of sterilised PBS and then centrifuged at $5000 \mathrm{~g}$ for $10 \mathrm{~min}$ The supernatant was discarded, and the pellet was stored at $-80^{\circ} \mathrm{C}$ until it was used for the extraction of RNA (or DNA). $\mathrm{RNA}^{(9,10)}$ and $\mathrm{DNA}^{(11)}$ were isolated using the method described elsewhere. Finally, the nucleic acid fraction was suspended in $1 \mathrm{ml}$ nuclease-free water (Ambion, Inc.)

Determination of the bacterial count by RT-quantitative $P C R$. A standard curve was generated using RT-quantitative PCR data (using the threshold cycle, the cycle number when threshold fluorescence was reached) and the corresponding cell count, which was determined microscopically with 4,6diamidino-2-phenylindole (Vector Laboratories, Burlingame, CA, USA) staining ${ }^{(12)}$ for the dilution series of the standard strains described elsewhere ${ }^{(9,10)}$. To determine the bacteria present in samples, three serial dilutions of an extracted RNA sample were used for RT-quantitative PCR, and the threshold cycle values in the linear range of the assay were applied to the standard curve generated in the same experiment to obtain the corresponding bacterial cell count in each nucleic acid sample and then converted to the number of bacteria per sample. The specificity of the RT-quantitative PCR assay using group- or species-specific primers was determined as described previously ${ }^{(9,10)}$. The detection of methicillinresistant Staphylococcus aureus, methicillin-resistant coagulase-negative Staphylococcus, methicillin-sensitive S. aureus and methicillin-sensitive coagulase-negative Staphylococcus has all been described previously ${ }^{(13)}$. Quantitative analysis of LCS has been described previously ${ }^{(11)}$.

Measurements of faecal organic acid concentrations and $\mathrm{pH}$. A portion of the homogenised stool was isolated, weighed, mixed with $0 \cdot 15 \mathrm{M}$-perchloric acid in a fourfold volume and reacted at $4^{\circ} \mathrm{C}$ for $12 \mathrm{~h}$. Next, the mixture was centrifuged at $4^{\circ} \mathrm{C}$ at $20000 \mathrm{~g}$ for $10 \mathrm{~min}$, and the supernatant was filtered with a $0.45 \mu \mathrm{m}$ membrane filter (Millipore Japan, 
Tokyo, Japan) and sterilised. The concentrations of organic acids in this sample were measured using a Waters HPLC system (Waters 432 Conductive Detector; Waters Corporation, Milford, MA, USA) and a Shodex Rspack KC-811 column (Showa Denko, Tokyo, Japan) ${ }^{(14)}$. We prepared a standard mixed solution consisting of 1-20 mm-succinic acid, lactic acid, formic acid, acetic acid, propionic acid, isobutyric acid, butyric acid, isovaleric acid and valeric acid, and calculated the concentrations of organic acids based on the standard curve.

The stool $\mathrm{pH}$ was measured by directly inserting the glass electrode of a D-51 pH meter (Horiba Seisakusho Company Limited, Tokyo, Japan) into the homogenised stool.

\section{Statistical analysis}

The results of a 1-month survey in December 2006 were used in the analysis of the survey items in the medical record data. The statistical significance of the LcS-fermented milk-administered group was assessed against the nonadministered group as follows: Wilcoxon's rank sum test, which is a non-parametric alternative to the two-sample test, was used to assess the statistical significance in terms of the number of days that a fever persisted, while Fisher's exact test was used to analyse the prevalence of patients with a fever and infectious gastroenteritis. The average number of bacteria was analysed using the paired $t$ test. The bacterial detection rate was analysed using Fisher's direct probability test. We employed SPSS version 11 software (SPSS Japan, Inc., Tokyo, Japan). $P<0.05$ was regarded as significant.

\section{Results}

Prevalence of patients with norovirus gastroenteritis in December 2006

During the 1-month period from 1 to 31 December 2006, twenty-one cases of norovirus gastroenteritis were confirmed among thirty-eight subjects in the non-administered group (55\%). During the same period, there were twenty-seven cases of infectious gastroenteritis among thirty-nine subjects in the LcS-fermented milk-administered group (69\%). Therefore, there was no significant difference between the two

Table 2. Effect of probiotic-fermented milk beverage containing Lactobacillus casei strain Shirota on faecal microflora in the elderly persons (Mean values ( $\log _{10}$ cells) and standard deviations (per g of faeces))

\begin{tabular}{|c|c|c|c|c|c|c|c|c|c|}
\hline \multirow[b]{2}{*}{ Organisms } & \multicolumn{3}{|c|}{ Before ingestion } & \multicolumn{3}{|c|}{1 month of ingestion } & \multicolumn{3}{|c|}{2 months of ingestion } \\
\hline & Mean & SD & $n / n \dagger$ & Mean & SD & $n / n \dagger$ & Mean & SD & $n / n \dagger$ \\
\hline Total bacteria & $10 \cdot 5$ & 0.4 & $10 / 10$ & $10 \cdot 5$ & 0.4 & $10 / 10$ & $10 \cdot 4$ & 0.4 & $10 / 10$ \\
\hline \multicolumn{10}{|l|}{ Anaerobes } \\
\hline Clostridium leptum subgroup & $9 \cdot 7$ & 0.4 & $10 / 10$ & $9 \cdot 8$ & 0.4 & $10 / 10$ & $9 \cdot 5$ & 0.4 & $10 / 10$ \\
\hline C. coccoides group & $9 \cdot 3$ & 0.5 & $10 / 10$ & $9 \cdot 4$ & 0.3 & $10 / 10$ & $9 \cdot 1$ & 0.4 & $10 / 10$ \\
\hline Bacteroides fragilis group & $10 \cdot 2$ & 0.6 & $10 / 10$ & $10 \cdot 1$ & 0.6 & $10 / 10$ & 9.9 & 0.9 & $10 / 10$ \\
\hline Bifidobacterium & $8 \cdot 7$ & 0.9 & $10 / 10$ & $9 \cdot 3^{*}$ & 0.5 & $10 / 10$ & $9 \cdot 3^{* *}$ & 0.4 & $10 / 10$ \\
\hline Atopobium cluster & $9 \cdot 1$ & 0.3 & $9 / 10$ & $9 \cdot 2$ & 0.6 & $9 / 10$ & $8 \cdot 8$ & 0.6 & $10 / 10$ \\
\hline Prevotella & $7 \cdot 0$ & $1 \cdot 4$ & $6 / 10$ & $7 \cdot 0$ & 0.8 & $5 / 10$ & $6 \cdot 4$ & $1 \cdot 2$ & $5 / 10$ \\
\hline C. perfringens & $5 \cdot 3$ & $1 \cdot 0$ & $7 / 10$ & $5 \cdot 1$ & $1 \cdot 8$ & $7 / 10$ & $4 \cdot 3$ & $1 \cdot 2$ & $7 / 10$ \\
\hline C. difficile & 4.9 & 0.8 & $8 / 10$ & $4 \cdot 8$ & $1 \cdot 3$ & $6 / 10$ & $4 \cdot 0^{\star}$ & & $2 / 10 \ddagger$ \\
\hline \multicolumn{10}{|l|}{ Facultative anaerobes } \\
\hline Total Lactobacillus & $7 \cdot 4$ & $1 \cdot 3$ & $10 / 10$ & $8 \cdot 3^{\star *}$ & 0.4 & $10 / 10$ & $8 \cdot 2$ & 0.4 & $10 / 10$ \\
\hline L. acidophilus subgroup & 6.5 & $1 \cdot 2$ & $10 / 10$ & 6.4 & 1.9 & $10 / 10$ & 6.9 & $1 \cdot 0$ & $8 / 10$ \\
\hline L. brevis & $<2 \cdot 1$ & & $0 / 10$ & $<2 \cdot 1$ & & $0 / 10$ & $<2 \cdot 1$ & & $0 / 10$ \\
\hline L. casei subgroup & $5 \cdot 3$ & $1 \cdot 4$ & $8 / 10$ & $8 \cdot 1^{\star *}$ & 0.3 & $10 / 10$ & $8 \cdot 1^{\star *}$ & 0.4 & $10 / 10$ \\
\hline L. fermentum & $6 \cdot 7$ & $1 \cdot 2$ & $7 / 10$ & $6 \cdot 0$ & 0.8 & $6 / 10$ & $5 \cdot 5$ & 0.9 & $8 / 10$ \\
\hline L. fructivorans & $<3.0$ & & $0 / 10$ & $<3.0$ & & $0 / 10$ & $<3.0$ & & $0 / 10$ \\
\hline L. plantarum subgroup & 3.3 & $1 \cdot 4$ & $8 / 10$ & $3 \cdot 0$ & 0.6 & $6 / 10$ & 2.5 & 0.4 & $6 / 10$ \\
\hline L. reuteri subgroup & $5 \cdot 5$ & $2 \cdot 1$ & $9 / 10$ & $4 \cdot 7$ & $2 \cdot 0$ & $10 / 10$ & $5 \cdot 0$ & $1 \cdot 7$ & $9 / 10$ \\
\hline L. ruminis subgroup & $6 \cdot 0$ & $2 \cdot 2$ & $9 / 10$ & $5 \cdot 4$ & $2 \cdot 1$ & $9 / 10$ & $6 \cdot 2$ & $2 \cdot 0$ & $6 / 10$ \\
\hline L. sakei subgroup & $3 \cdot 7$ & 0.4 & $9 / 10$ & 3.7 & 0.4 & $5 / 10$ & 2.7 & 1.5 & $5 / 10$ \\
\hline Enterobacteriaceae & $7 \cdot 9$ & 0.6 & $10 / 10$ & $7 \cdot 4$ & $1 \cdot 0$ & $10 / 10$ & $7 \cdot 5^{\star}$ & 0.3 & $10 / 10$ \\
\hline Enterococcus & $7 \cdot 4$ & $1 \cdot 0$ & $10 / 10$ & $7 \cdot 1$ & 0.8 & $10 / 10$ & $6 \cdot 8$ & $1 \cdot 1$ & $10 / 10$ \\
\hline Staphylococcus & $5 \cdot 2$ & 0.7 & $10 / 10$ & $4 \cdot 8$ & 0.5 & $10 / 10$ & 4.9 & 0.8 & $10 / 10$ \\
\hline MRSA & $<4.0$ & & $0 / 10$ & $<4.0$ & & $0 / 10$ & $<4.0$ & & $0 / 10$ \\
\hline MRCNS & $<4.0$ & & $0 / 10$ & $<4.0$ & & $0 / 10$ & $<4.0$ & & $0 / 10$ \\
\hline MSSA & 4.7 & & $1 / 10$ & $<4.0$ & & $0 / 10$ & $<4.0$ & & $0 / 10$ \\
\hline MSCNS & $5 \cdot 2$ & 0.7 & $10 / 10$ & 4.8 & 0.5 & $10 / 10$ & 4.9 & 0.8 & $10 / 10$ \\
\hline \multicolumn{10}{|l|}{ Aerobes } \\
\hline Pseudomonas & $3 \cdot 3$ & 1.0 & $8 / 10$ & $4 \cdot 0$ & 0.5 & $3 / 10$ & $3 \cdot 3^{*}$ & & $2 / 10 \ddagger$ \\
\hline \multicolumn{10}{|l|}{ Ingested bacteria } \\
\hline L. casei strain Shirota & $<5.5$ & & $0 / 10$ & $8 \cdot 0$ & 0.3 & $10 / 10$ & $8 \cdot 0$ & 0.4 & $10 / 10$ \\
\hline
\end{tabular}

MRSA, methicillin-resistant Staphylococcus aureus; MRCNS, methicillin-resistant coagulase-negative Staphylococcus; MSSA, methicillin-sensitive S. aureus; MSCNS, methicillinsensitive coagulase-negative Staphylococcus.

Mean values were significantly different between before ingestion and during ingestion (Student's $t$ test (paired)): ${ }^{\star} P<0.05 ;{ }^{\star \star} P<0.01$.

$\dagger$ The number of samples in which the bacteria were detected/the number of samples examined. 
groups. There was also no significant difference in terms of the number of days on which diarrhoea and vomiting were observed (data not shown).

Number of days that a fever of at least 37 and $38^{\circ} \mathrm{C}$ was observed after contracting infectious gastroenteritis caused by norovirus in December 2006

The number of days in October and November 2006 on which a fever of at least $37^{\circ} \mathrm{C}$ was observed was compared between the LcS-fermented milk-administered and non-administered groups (Table 1). The results for October 2006 were 1.0 (SD $2 \cdot 8) \mathrm{d}$ for the LcS-fermented milk-administered group (thirtynine subjects) and 1.0 (SD 1.9) $\mathrm{d}$ for the non-administered group (thirty-eight subjects). The number of days in November 2006 was found to be 1.0 (SD 1.9) $\mathrm{d}$ for the LCS-fermented milk-administered group (thirty-nine subjects) and 0.4 (SD 0.8) $\mathrm{d}$ for the non-administered group (thirty-eight subjects). Based on the verification that there had been no significant difference between the two groups in terms of the number of days that a fever of at least $37^{\circ} \mathrm{C}$ was observed in October and November, the data for December were analysed. The number of days on which a fever of at least $37^{\circ} \mathrm{C}$ was observed after the subjects contracted infectious gastroenteritis in the non-administered group was $2.9(\mathrm{SD} 2.3) \mathrm{d}$, and that in the LcS-fermented milk-administered group was 1.5 (SD 1.7) d, showing that as a result of LcS-fermented milk intake, the number of days of fever was significantly reduced $(P<0 \cdot 05$; Table 1). Furthermore, compared with the non-administered group, there were fewer days of fever of at least $38^{\circ} \mathrm{C}$ in the LcS-fermented milk-administered group during the same period ( $P=0 \cdot 09$; Table 1$)$.

Effect of Lactobacillus casei strain Shirota-fermented milk beverage on the faecal microflora

Comparing the pre-ingestion data and those taken 1 month after ingestion had started, while there was no significant difference in terms of the total number of bacteria in the faecal samples, the levels of Bifidobacterium, total Lactobacillus and the $L$. casei subgroup had increased significantly ( $P<0.05, P<0.01$ and $P<0.01$, respectively; Table 2$)$. The data taken 2 months after ingestion had started also indicated that the population levels of Bifidobacterium and the $L$. case $i$ subgroup had significantly increased $(P<0.01$ each). On the other hand, compared with the pre-ingestion data, the data taken 2 months after ingestion had started showed that the level of Enterobacteriaceae had significantly decreased $(P<0.05)$. There was also a significant decrease in the rate of detected Pseudomonas $(P<0.05)$. Furthermore, during the ingestion period, no methicillin-resistant $S$. aureus or methicillin-resistant coagulase-negative Staphylococcus was detected (Table 2).

The bacterium LcS, the bacteria contained in the test diet, had never been detected in faecal samples taken from residents before LcS-fermented milk ingestion; however, it was detected in all subjects during ingestion, with the bacterial count of a faecal sample being $10^{7}-10^{8}$ cells/g (Table 2 ).

\section{Effect of Lactobacillus casei strain Shirota-fermented milk beverage on the concentration of faecal organic acids}

Compared with the pre-ingestion data, the concentrations of total organic acids and acetic acid were significantly higher in faecal samples taken 1 month after ingestion had started $(P<0.05$ and $P<0.01$, respectively; Table 3$)$ and were even higher in faecal samples taken 2 months after ingestion had started $(P<0.05$ each). Compared with the pre-ingestion data, faecal $\mathrm{pH}$ was significantly lower in samples taken 1 and 2 months after ingestion had started $(P<0 \cdot 01$ each; Table 3).

\section{Correlations between faecal Bifidobacterium and acetic acid concentrations}

The correlation between the population of faecal bacteria and the organic acid concentration in all thirty faecal samples collected from ten subjects before the ingestion of LcS-fermented

Table 3. Effect of probiotic-fermented milk beverage containing Lactobacillus casei strain Shirota on the concentration of organic acids in the elderly persons

(Mean values ( $\mu \mathrm{mol})$ and standard deviations (per g faeces))

\begin{tabular}{|c|c|c|c|c|c|c|c|c|c|}
\hline \multirow[b]{2}{*}{ Item } & \multicolumn{3}{|c|}{ Before ingestion } & \multicolumn{3}{|c|}{1 month of ingestion } & \multicolumn{3}{|c|}{2 months of ingestion } \\
\hline & Mean & SD & $n / n \dagger$ & Mean & SD & $n / n \dagger$ & Mean & SD & $n / n \dagger$ \\
\hline Total organic acids & $71 \cdot 0$ & 38.7 & $10 / 10$ & $97 \cdot 3^{*}$ & $17 \cdot 1$ & $10 / 10$ & $110 \cdot 5^{\star}$ & 8.9 & $10 / 10$ \\
\hline Succinic acid & 0.1 & & $1 / 10$ & $5 \cdot 1$ & 1.6 & $5 / 10$ & $3 \cdot 1$ & $2 \cdot 0$ & $5 / 10$ \\
\hline Lactic acid & 3.6 & & $1 / 10$ & $<0.2$ & & $0 / 10$ & $1 \cdot 2$ & & $1 / 10$ \\
\hline Formic acid & $<0.05$ & & $0 / 10$ & $<0.05$ & & $0 / 10$ & $1 \cdot 1$ & 1.0 & $7 / 10$ \\
\hline Acetic acid & $50 \cdot 0$ & $24 \cdot 7$ & $10 / 10$ & $68 \cdot 6^{\star \star}$ & $17 \cdot 0$ & $10 / 10$ & $75 \cdot 9^{*}$ & $12 \cdot 6$ & $10 / 10$ \\
\hline Propionic acid & $12 \cdot 0$ & 6.9 & $10 / 10$ & $12 \cdot 8$ & $2 \cdot 3$ & $10 / 10$ & $15 \cdot 9$ & $4 \cdot 8$ & $10 / 10$ \\
\hline Butyric acid & $7 \cdot 9$ & $5 \cdot 1$ & $7 / 10$ & $11 \cdot 3$ & 1.9 & $10 / 10$ & $12 \cdot 3$ & $5 \cdot 8$ & $10 / 10$ \\
\hline Isovaleric acid & 3.4 & $3 \cdot 6$ & $7 / 10$ & 4.9 & $2 \cdot 6$ & $4 / 10$ & 3.4 & $1 \cdot 3$ & $8 / 10$ \\
\hline Valeric acid & 3.9 & & $2 / 10$ & $<0.8$ & & $0 / 10$ & 1.6 & 0.8 & $3 / 10$ \\
\hline $\mathrm{pH}$ & $7 \cdot 2$ & 0.3 & $10 / 10$ & $6 \cdot 8^{\star *}$ & 0.3 & $10 / 10$ & $6 \cdot 8^{\star *}$ & 0.4 & $10 / 10$ \\
\hline
\end{tabular}

Mean values were significantly different between before ingestion and during ingestion (Student's $t$ test (paired)): ${ }^{*} P<0.05 ;{ }^{\star *} P<0.01$. $\dagger$ The number of samples in which the organic acids were detected/the number of samples examined. 


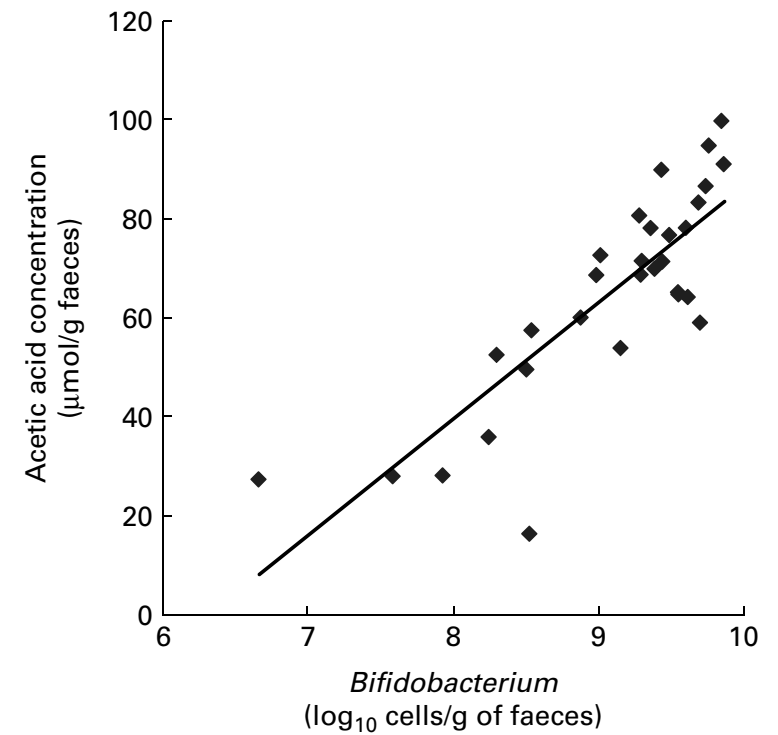

Fig. 2. Pearson's correlation between faecal Bifidobacterium and acid concentrations $\quad(P<0.001) . \quad n \quad 30 ; \quad y=23.537 x-148.78 ; \quad R^{2} \quad 0.6904$; $P=1.317 \times 10^{-8}$.

milk as well as 1 and 2 months after ingestion had started was analysed. A positive correlation was shown between the number of Bifidobacterium and acetic acid concentration $\left(P=1.32 \times 10^{-8}\right)($ Fig. 2)

\section{Discussion}

Norovirus is the most common cause of infectious gastroenteritis. In recent years, many incidences of norovirus gastroenteritis have been reported ${ }^{(15-17)}$. According to a report on gastroenteritis by the Centers for Disease Control and Prevention in the USA, most cases of infectious gastroenteritis caused by norovirus occurred at elderly welfare facilities ${ }^{(18)}$. In infectious gastroenteritis casued by norovirus, fever is one indicator that the disease may become severe. It may also increase the risk of aspiration pneumonia, leading to a deterioration in the general condition of patients ${ }^{(19)}$. As such, in elderly care facilities, the prevention and management of norovirus gastroenteritis has thus become extremely important. No preventive measures were being taken for residents of the Municipal Health Service Facility for the Aged, who were provided with LcS-fermented milk from April 2006 onwards, against norovirus gastroenteritis, outbreaks of which occurred in December 2006. However, it was found that the duration of fever (at least 37 and $38^{\circ} \mathrm{C}$ ) after the onset of infectious gastroenteritis caused by norovirus was significantly shorter. In general, fever is not a specific indicator of norovirus gastroenteritis. However, it is important to shorten the duration of fever because a persistent fever can contribute to dehydration, which is one of the most serious complications in elderly people presenting with norovirus gastroenteritis $^{(20,21)}$.

Furthermore, the acetic acid concentration rose in faecal samples taken from residents after the intake of LcS-containing fermented milk, while the $\mathrm{pH}$ decreased. Acetic acid, the most common SCFA in the colon, has been reported to demonstrate in vitro bactericidal activity against pathogenic bacteria $^{(22,23)}$, but not against viruses. As such, acetic acid in the colon is believed to have weak, direct bacteriostatic activity, so it is believed that the intake of LcS-fermented milk would not have prevented infectious gastroenteritis caused by norovirus.

On the other hand, acetic acid and other SCFA in the colon are associated not only with water and salt absorption but also with gastrointestinal tract motility. These constitute the main important energetic substrate for epithelial cells in the intestine and also serve as an energy source for the entire body through portal blood flow and the liver ${ }^{(24)}$. It has been reported that natural killer cell activity in the blood in healthy adults would significantly improve with the intake of LcS-fermented milk, probably via IL-12 production by monocytes/ macrophages in response to $\mathrm{LcS}^{(2)}$. It has also been reported that the continuous intake of LcS-fermented milk would alleviate symptoms of spastic spinal paralysis, which is peculiar to human $\mathrm{T}$ lymphotropic virus type 1-associated myelopathy patients ${ }^{(25)}$, who have weakened natural defences, and it is believed that the onset of symptoms is related to a decline in immunocompetence. Natural killer cell activity in human $\mathrm{T}$ lymphotropic virus type 1 -associated myelopathy patients who drank LcS-fermented milk on a continuous basis significantly improved, and it is believed that the recovery of natural defences contributed to the alleviation of symptoms. It has therefore been suggested that the main factor for the activity of LcS-fermented milk in suppressing fever associated with norovirus gastroenteritis could be increased disease resistance, such as an improved intestinal environment and immunoenhancing effects.

We did not perform faecal microflora analyses on norovirus gastroenteritis patients and then compare the findings with those for non-infected subjects in the present study. There have so far been no studies made on intestinal floral abnormalities in norovirus gastroenteritis, nor are there any available references regarding the effect of probiotics on acute gastroenteritis except for rotavirus infection ${ }^{(26-28)}$. Prophylactic use of Lactobacillus GG significantly reduced the risk of nosocomial rotavirus gastroenteritis in children ${ }^{(26)}$. Other studies have shown Lactobacillus GG to reduce the duration of symptoms and viral excretion, while promoting a recovery from acute diarrhoea in children with rotavirus gastroenteritis ${ }^{(27,28)}$.

In the present study, the intestinal flora and environment of the residents of the investigated facility were disturbed, and the possibility that such residents may have been highly susceptible to harmful bacteria in the intestine is strongly suggested; however, with the intake of LcS-fermented milk, the population of Bifidobacteria increased, which significantly optimised the intestinal environment, thereby limiting the growth of harmful bacteria in the intestine based on the findings of previous experimental and clinical studies ${ }^{(3,29-33)}$. Furthermore, a correlation between the increase in the Bifidobacterium population numbers in the intestine and the rise in acetic acid concentration was observed (Fig. 2). It has been reported that acetic acid, the main metabolite produced 
by Bifidobacterium, demonstrates strong in vitro bactericidal activity against Enterobacteriaceae ${ }^{(23)}$, and that the bactericidal activity of acetic acid is exerted under non-dissociating conditions $^{(22)}$. In consideration of these findings, it is believed that improvement of the intestinal environment associated with an increase in the Bifidobacterium population, such as a rise in acetic acid concentration and a decrease in the $\mathrm{pH}$, is important in limiting the overgrowth of harmful bacteria in the intestine.

Although the continuous intake of LcS-fermented milk did not prevent infectious gastroenteritis caused by norovirus among the frail, long-term elderly residents of the Municipal Health Service Facility for the Aged, it was found that the fever associated with gastroenteritis was alleviated, and that the internal flora and environment were optimised. It is therefore suggested that the continuous intake of LCS-fermented milk helps the residents of elderly care facilities to recover from norovirus gastroenteritis.

\section{Acknowledgements}

We wish to acknowledge and extend our sincere gratitude to Ms Yuko Hasegawa and other staff at 'Yuyu' Municipal Health Service Facility for the Aged, for their cooperation in the present study. We also wish to express our sincere gratitude for the instruction on statistical analysis by Dr Hiroshi Nagata, as well as for the support of bacterial flora analysis by Mr Norikatsu Yuki and Mr Akira Takahashi, of Yakult Central Institute for Microbiological Research. The authors report no conflicts of interest. No financial assistance was received in support of the study. All authors contributed equally for intellectual input and writing of the manuscript.

\section{References}

1. Reid G (2005) Food and Agricultural Organization of the United Nation and the WHO. The importance of guidelines in the development and application of probiotics. Curr Pharm 11, 11-16.

2. Takeda K, Suzuki T, Shimada SI, et al. (2006) Interleukin-12 involved in the enhancement of human natural killer cell activity by Lactobacillus casei Shirota. Clin Exp Immunol 146, 109-115.

3. Sugawara G, Nagino M, Nishio H, et al. (2006) Perioperative synbiotic treatment to prevent postoperative infectious complications in biliary cancer surgery: a randomized controlled trial. Ann Surg 244, 706-714.

4. Aso Y, Akaza H, Kotake T, et al. (1995) Preventive effect of a Lactobacillus casei preparation on the recurrence of superficial bladder cancer in a double-blind trial. The BLP Study Group. Eur Urol 27, 104-109.

5. Tamura M, Shikina T, Morihana T, et al. (2007) Effects of probiotics on allergic rhinitis induced by Japanese cedar pollen: randomized double-blind, placebo-controlled clinical trial. Int Arch Allergy Immunol 143, 75-82.

6. Shioiri T, Yahagi K, Nakayama S, et al. (2006) The effects of synbiotic fermented milk beverage containing Lactobacillus casei strain Shirota and transgalactosylated oligosaccharides on defecation frequency, intestinal microflora, organic acid concentrations, and putrefactive metabolites of sub-optimal health state volunteers: a randomized placebo-controlled cross-over study. Biosci Microflora 25, 137-146.

7. Takeda K \& Okumura K (2007) Effects of a fermented milk drink containing Lactobacillus casei strain Shirota on the human NK-cell activity. J Nutr 137, 791S-793S.

8. Matsumoto K, Takada T, Shimizu K, et al. (2006) The effects of a probiotic milk product containing Lactobacillus casei strain Shirota on the defecation frequency and the intestinal microflora of sub-optimal health state volunteers: a randomized placebo-controlled cross-over study. Biosci Microflora 25, 39-48.

9. Matsuda K, Tsuji H, Asahara T, et al. (2007) Sensitive quantitative detection of commensal bacteria by rRNA-targeted reverse transcription-PCR. Appl Environ Microbiol 73, 32-39.

10. Matsuda K, Tsuji H, Asahara T, et al. (2009) Establishment of an analytical system for the human fecal microbiota, based on reverse transcription-quantitative PCR targeting of multicopy rRNA molecules. Appl Environ Microbiol $\mathbf{7 5}$ 1961-1969.

11. Fujimoto J, Matsuki T, Sasamoto M, et al. (2008) Identification and quantification of Lactobacillus casei strain Shirota in human feces with strain-specific primers derived from randomly amplified polymorphic DNA. Int J Food Microbiol 126, 210-215.

12. Jansen GJ, Wildeboer-Veloo AC, Tonk RH, et al. (1999) Development and validation of an automated, microscopybased method for enumeration of groups of intestinal bacteria. J Microbiol Methods 37, 215-221.

13. Wada M, Enkhtuya L, Bian L, et al. (2010) Quantitative reverse transcription-PCR assay for rapid detection of methicillin-resistant Staphylococcus aureus. J Appl Microbiol 108, 779-788.

14. Kamiya S, Nagino M, Kanazawa H, et al. (2004) The value of bile replacement during external biliary drainage: an analysis of intestinal permeability, integrity, and microflora. Ann Surg 239, 510-517.

15. Centers for Disease Control and Prevention (2009) Recurring norovirus outbreaks in a long-term residential treatment facility - Oregon, 2007. MMWR Morb Mortal Wkly Rep 58 694-698.

16. Navarro G, Sala RM, Segura F, et al. (2005) An outbreak of norovirus infection in a long-term-care unit in Spain. Infect Control Hosp Epidemiol 26, 259-262.

17. Siebenga JJ, Vennema H, Zheng DP, et al. (2009) Norovirus illness is a global problem: emergence and spread of norovirus GII.4 variants, 2001-2007. J Infect Dis 200, 802-812.

18. Fankhauser RL, Monroe SS, Noel JS, et al. (2002) Epidemiologic and molecular trends of "Norwalk-like viruses" associated with outbreaks of gastroenteritis in the United States. J Infect Dis 186, 1-7.

19. Honma S (2002) Characteristic of Norwalk gastroenteritis and its prevention. Nippon Rinsho 60, 1202-1207.

20. Pals JK, Weinberg AD, Beal LF, et al. (1995) Clinical triggers for detection of fever and dehydration. Implications for longterm care nursing. J Gerontol Nurs 21, 13-19.

21. Weinberg AD, Pals JK, Levesque PG, et al. (1994) Dehydration and death during febrile episodes in the nursing home. J Am Geriatr Soc 42, 968-971.

22. Eklund $\mathrm{T}$ (1983) The antimicrobial effect of dissociated and undissociated sorbic acid at different pH levels. J Appl Bacteriol 54, 383-389.

23. Ostling CE \& Lindgren SE (1993) Inhibition of enterobacteria and Listeria growth by lactic, acetic and formic acids. $J$ Appl Bacteriol 75, 18-24. 
24. Sakata T (1997) Influence of short chain fatty acids on intestinal growth and functions. Adv Exp Med Biol 427, 191-199.

25. Matsuzaki T, Saito M, Usuku K, et al. (2005) A prospective uncontrolled trial of fermented milk drink containing viable Lactobacillus casei strain Shirota in the treatment of HTLV-1 associated myelopathy/tropical spastic paraparesis. I Neurol Sci 237, 75-81.

26. Szajewska H, Kotowska M, Mrukowicz JZ, et al. (2001) Efficacy of Lactobacillus GG in prevention of nosocomial diarrhea in infants. J Pediatr 138, 361-365.

27. Isolauri E, Juntunen M, Rautanen T, et al. (1991) A human Lactobacillus strain (Lactobacillus casei sp. strain GG) promotes recovery from acute diarrhea in children. Pediatrics 88, 90-97.

28. Guarino A, Canani RB, Spagnuolo MI, et al. (1997) Oral bacterial therapy reduces the duration of symptoms and of viral excretion in children with mild diarrhea. J Pediatr Gastroenterol Nutr 25, 516-519.

29. Asahara T, Nomoto K, Shimizu K, et al. (2001) Increased resistance of mice to Salmonella enterica serovar Typhimurium infection by synbiotic administration of bifidobacteria and transgalactosylated oligosaccharides. J Appl Microbiol 91, 985-996.

30. Asahara T, Shimizu K, Nomoto K, et al. (2001) Antibacterial effect of fermented milk containing Bifidobacterium breve, Bifidobacterium bifidum and Lactobacillus acidophilus against indigenous Escherichia coli infection in mice. Microb Ecol Health Dis 13, 16-24.

31. Kanamori Y, Sugiyama M, Hashizume K, et al. (2004) Experience of long-term synbiotic therapy in seven short bowel patients with refractory enterocolitis. J Pediatr Surg 39, $1686-1692$.

32. Kanazawa H, Nagino M, Kamiya S, et al. (2005) Synbiotics reduce postoperative infectious complications: a randomized controlled trial in biliary cancer patients undergoing hepatectomy. Langenbecks Arch Surg 390, 104-113.

33. Shimizu K, Ogura H, Goto M, et al. (2009) Synbiotics decrease the incidence of septic complications in patients with severe SIRS: a preliminary report. Dig Dis Sci 54, 1071-1078. 